\title{
Erratum: Properties of a New Group of Cosmic Nuclei: Results from the Alpha Magnetic Spectrometer on Sodium, Aluminum, and Nitrogen [Phys. Rev. Lett. 127, 021101 (2021)]
}

M. Aguilar, L. Ali Cavasonza, B. Alpat, G. Ambrosi, L. Arruda, N. Attig, F. Barao, L. Barrin, A. Bartoloni, S. Başeğmez-du Pree, R. Battiston, M. Behlmann, B. Beranek, J. Berdugo, B. Bertucci, V. Bindi, K. Bollweg, B. Borgia, M. J. Boschini, M. Bourquin, E. F. Bueno, J. Burger, W. J. Burger, S. Burmeister, X. D. Cai, M. Capell, J. Casaus, G. Castellini, F. Cervelli, Y. H. Chang, G. M. Chen, G. R. Chen, H. S. Chen, Y. Chen, L. Cheng, H. Y. Chou, S. Chouridou, V. Choutko, C. H. Chung, C. Clark, G. Coignet, C. Consolandi, A. Contin, C. Corti, Z. Cui, K. Dadzie, C. Delgado, S. Della Torre, M. B. Demirköz, L. Derome, S. Di Falco, V. Di Felice, C. Díaz, F. Dimiccoli, P. von Doetinchem, F. Dong,

F. Donnini, M. Duranti, A. Egorov, A. Eline, J. Feng, E. Fiandrini, P. Fisher, V. Formato, C. Freeman, C. Gámez, R. J. García-López, C. Gargiulo, H. Gast, M. Gervasi, F. Giovacchini, D. M. Gómez-Coral, J. Gong, C. Goy, V. Grabski, D. Grandi, M. Graziani, S. Haino, K. C. Han, R. K. Hashmani, Z. H. He, B. Heber, T. H. Hsieh, J. Y. Hu, M. Incagli, W. Y. Jang, Yi Jia, H. Jinchi, B. Khiali, G. N. Kim, Th. Kirn, M. Konyushikhin, O. Kounina, A. Kounine, V. Koutsenko, D. Krasnopevtsev, A. Kuhlman, A. Kulemzin, G. La Vacca, E. Laudi, G. Laurenti, I. Lazzizzera, A. Lebedev, H. T. Lee, S. C. Lee, J. Q. Li, M. Li, Q. Li, S. Li, J. H. Li, Z. H. Li, J. Liang, C. Light, C. H. Lin, T. Lippert, J. H. Liu, Z. Liu, S. Q. Lu, Y. S. Lu, K. Luebelsmeyer, J. Z. Luo, Xi Luo, F. Machate, C. Mañá, J. Marín, J. Marquardt, T. Martin, G. Martínez, N. Masi, D. Maurin, T. Medvedeva, A. Menchaca-Rocha, Q. Meng, V. V. Mikhailov, M. Molero, P. Mott, L. Mussolin, J. Negrete, N. Nikonov, F. Nozzoli, A. Oliva, M. Orcinha, M. Palermo, F. Palmonari, M. Paniccia, A. Pashnin, M. Pauluzzi, S. Pensotti, H. D. Phan, V. Plyaskin, M. Pohl, S. Poluianov, X. Qin, Z. Y. Qu, L. Quadrani, P. G. Rancoita, D. Rapin, A. Reina Conde, E. Robyn, S. Rosier-Lees, A. Rozhkov, D. Rozza, R. Sagdeev, S. Schael, A. Schulz von Dratzig, G. Schwering, E. S. Seo, Z. Shakfa, B. S. Shan, T. Siedenburg, C. Solano, J. W. Song, X. J. Song, R. Sonnabend,

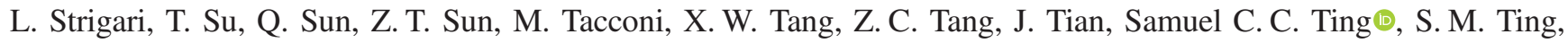
N. Tomassetti, J. Torsti, C. Tüysüz, T. Urban, I. Usoskin, V. Vagelli, R. Vainio, M. Valencia-Otero, E. Valente, E. Valtonen, M. Vázquez Acosta, M. Vecchi, M. Velasco, J. P. Vialle, C. X. Wang, L. Wang, L. Q. Wang, N. H. Wang, Q. L. Wang, S. Wang, X. Wang, Yu Wang, Z. M. Wang, J. Wei, Z. L. Weng, H. Wu, R. Q. Xiong, W. Xu, Q. Yan, Y. Yang, I. I. Yashin, H. Yi, Y. M. Yu, Z. Q. Yu, M. Zannoni, C. Zhang, F. Zhang, F. Z. Zhang, J. H. Zhang, Z. Zhang, F. Zhao, C. Zheng, Z. M. Zheng, H. L. Zhuang, V. Zhukov, A. Zichichi, and P. Zuccon

\section{(AMS Collaboration)}

(Received 23 August 2021; published 8 October 2021)

DOI: 10.1103/PhysRevLett.127.159901

After publication of our Letter, we learned that we have omitted citations to Refs. [1,2] below. These publications provide model-dependent results on the nuclei flux ratios at the source from low-energy measurements. Our Letter, Table I, provides model-independent results at the source for $\mathrm{N} / \mathrm{O}, \mathrm{Na} / \mathrm{Si}$, and $\mathrm{Al} / \mathrm{Si}$ over the wide energy range $\sim 6 \mathrm{GV}$ to $3 \mathrm{TV}$.

The physics results and conclusions of our Letter remain the same.

We thank Dr. Martin H. Israel and Dr. Alan C. Cummings for pointing out this omission.

[1] M. H. Israel et al., Elemental composition at the cosmic-ray source derived from the ACE-CRIS instrument. I. ${ }_{6} \mathrm{C}$ to ${ }_{28} \mathrm{Ni}$, Astrophys. J. 865, 69 (2018).

[2] A. C. Cummings, E. C. Stone, B. C. Heikkila, N. Lal, W. R. Webber, G. Jóhannesson, I. V. Moskalenko, E. Orlando, and T. A. Porter, Galactic cosmic rays in the local interstellar medium: Voyager 1 observations and model results, Astrophys. J. 831, 18 (2016).

Published by the American Physical Society under the terms of the Creative Commons Attribution 4.0 International license. Further distribution of this work must maintain attribution to the author(s) and the published articles title, journal citation, and DOI. 